\title{
Risk of acute myocardial infarction with NSAIDs in real world use: bayesian meta-analysis of individual patient data
}

\author{
Michèle Bally, ${ }^{1,2}$ Nandini Dendukuri, ${ }^{3,4}$ Benjamin Rich, ${ }^{4}$ Lyne Nadeau, ${ }^{4}$ Arja Helin-Salmivaara, ${ }^{5}$ \\ Edeltraut Garbe, ${ }^{6}$ James M Brophy $2,4,7$
}

Department of Pharmacy and Research Centre, Centre hospitalier de l'Université de Montréal, Montreal, H2X 1N4, Canada

2Department of Epidemiology, Biostatistics and Occupationa Health, McGill University, Montreal, Canada

${ }^{3}$ Technology Assessment Unit of the McGill University Health

Centre, Montreal, Canada

${ }^{4}$ Division of Clinical

Epidemiology, McGill University Health Centre-Research

Institute, Montreal, Canada ${ }^{5}$ Unit of Primary Care, Hospital District of Helsinki and Uusimaa, Helsinki, Finland

${ }^{6}$ Department of Clinical Epidemiology, Leibniz Institute for Prevention Research and Epidemiology-BIPS, Bremen, Germany

7Department of Medicine, McGill University, Montreal, Canada Correspondence to: M Bally michele.bally.chum@ssss.gouv. qc.ca

Additional material is published online only. To view please visit the journal online.

Cite this as: BMJ 2017;357:j1909 http://dx.doi.org/10.1136/bmj.j1909

Accepted: 10 April 2017

\section{ABSTRACT}

OBJECTIVE

To characterise the determinants, time course, and risks of acute myocardial infarction associated with use of oral non-steroidal anti-inflammatory drugs (NSAIDs).

\section{DESIGN}

Systematic review followed by a one stage bayesian individual patient data meta-analysis.

DATA SOURCES

Studies from Canadian and European healthcare databases.

\section{REVIEW METHODS}

Eligible studies were sourced from computerised drug prescription or medical databases, conducted in the general or an elderly population, documented acute myocardial infarction as specific outcome, studied selective cyclo-oxygenase- 2 inhibitors (including rofecoxib) and traditional NSAIDs, compared risk of acute myocardial infarction in NSAID users with non-users, allowed for time dependent analyses, and minimised effects of confounding and

misclassification bias.

\section{EXPOSURE AND OUTCOMES}

Drug exposure was modelled as an indicator variable incorporating the specific NSAID, its recency, duration of use, and dose. The outcome measures were the summary adjusted odds ratios of first acute myocardial infarction after study entry for each category of NSAID use at index date (date of acute myocardial infarction for cases, matched date for controls) versus non-use in the preceding year and the posterior probability of acute myocardial infarction.

\section{WHAT IS ALREADY KNOWN ON THIS TOPIC}

Evidence suggests that both traditional and cyclo-oxygenase-2 selective nonsteroidal anti-inflammatory drugs (NSAIDs) can increase the risk of acute myocardial infarction

The timing of the risk, the effect of dose, treatment duration, and the comparative risks between NSAIDs are poorly understood

\section{WHAT THIS STUDY ADDS}

Using a bayesian meta-analysis of individual patient data and studying real world settings, it is shown that all traditional NSAIDs, including naproxen, appear to be associated with an increased risk of acute myocardial infarction

The risk with celecoxib does not seem to be greater than that with traditional NSAIDs. Onset of risk occurs in the first week

Short term use for 8-30 days at a high daily dose (celecoxib $>200 \mathrm{mg}$, diclofenac $>100 \mathrm{mg}$, ibuprofen $>1200 \mathrm{mg}$, and naproxen $>750 \mathrm{mg}$ ) is associated with the greatest harms, without obvious further increases in risk beyond the first 30 days

\section{RESULTS}

A cohort of 446763 individuals including 61460 with acute myocardial infarction was acquired. Taking any dose of NSAIDs for one week, one month, or more than a month was associated with an increased risk of myocardial infarction. With use for one to seven days the probability of increased myocardial infarction risk (posterior probability of odds ratio $>1.0$ ) was $92 \%$ for celecoxib, $97 \%$ for ibuprofen, and $99 \%$ for diclofenac, naproxen, and rofecoxib. The corresponding odds ratios (95\% credible intervals) were 1.24 (0.91 to 1.82) for celecoxib, 1.48 (1.00 to 2.26) for ibuprofen, 1.50 (1.06 to 2.04) for diclofenac, 1.53 (1.07 to 2.33) for naproxen, and 1.58 (1.07 to 2.17 ) for rofecoxib. Greater risk of myocardial infarction was documented for higher dose of NSAIDs. With use for longer than one month, risks did not appear to exceed those associated with shorter durations.

\section{CONCLUSIONS}

All NSAIDs, including naproxen, were found to be associated with an increased risk of acute myocardial infarction. Risk of myocardial infarction with celecoxib was comparable to that of traditional NSAIDS and was lower than for rofecoxib. Risk was greatest during the first month of NSAID use and with higher doses.

\section{Introduction}

It is generally accepted that oral non-steroidal anti-inflammatory drugs (NSAIDs) can increase the risk of acute myocardial infarction. Randomised controlled trials of NSAIDs have been of limited use for assessing this rare adverse event, as they had small cohorts and poor generalisability. ${ }^{12}$ The trials excluded those at highest cardiovascular risk or with established cardiovascular disease. ${ }^{34}$ Network meta-analyses of randomised controlled trials of NSAIDs and myocardial infarction risk have attempted to improve statistical power, but the results of direct and indirect comparisons of NSAIDs and placebo remain imprecise and occasionally inconclusive. ${ }^{34}$

The Prospective Randomized Evaluation of Celecoxib Integrated Safety vs. Ibuprofen Or Naproxen (PRECISION) trial was a large randomised controlled trial $(n=24081)$ that filled some of these knowledge gaps. This trial's conclusion of the non-inferiority of moderate dose celecoxib compared with ibuprofen and naproxen on a primary composite outcome of cardiovascular death, non-fatal myocardial infarction, or non-fatal stroke in patients with arthritis at moderate cardiovascular risk has challenged the convention that all selective cyclo-oxygenase-2 (COX 2) inhibitors share the same heightened cardiovascular risk as rofecoxib and that naproxen has superior cardiovascular safety. ${ }^{5}$ 
Although the PRECISION trial reported on myocardial infarction as a secondary outcome, it did not include a comparison with placebo and cannot inform on the comparative cardiovascular safety of NSAIDs other than as studied; this trial enrolled patients receiving standardised, fixed daily doses of NSAIDs for arthritis. ${ }^{5}$ Dosages and treatment duration in this and other NSAID randomised controlled trials ${ }^{34}$ may not represent the clinical reality of many patients who use these drugs in low or varying doses, or intermittently, and often switch between various NSAIDs. ${ }^{67}$ Risk of acute myocardial infarction associated with NSAIDs should be further characterised by pooling population based observational studies since these better reflect how NSAIDs are used in practice. ${ }^{8}$

We performed an individual patient data meta-analysis of studies from healthcare databases to determine the time course for risk of acute myocardial infarction and the effects of dose and of duration of continuous use for the main NSAIDs. The study was designed to capture the complex time varying nature of NSAID use. We verified that measurement of NSAID exposure was adequate for addressing the objectives of this study then examined various aspects of exposure that are relevant to the myocardial infarction outcome, including recency of use and the combined effect of dose and duration. To optimise the power to make useful clinical inferences we studied a large patient sample.

\section{Methods}

Systematic review

Two researchers developed literature search strategies and selected studies (MB, JMB). We searched Medline, Embase, and PubMed by applying filters for retrieval of observational studies and by combining these with the appropriate search terms for non-steroidal anti-inflammatory drugs (NSAIDs) and for myocardial infarction (see table 1 in web appendix 1). We also retrieved systematic reviews of non-randomised studies of cardiovascular adverse events associated with NSAIDs and manually searched their bibliography. To increase sensitivity for the myocardial infarction outcome, we included broader terms for cardiac morbidity. After an initial search, we set up weekly updates from June 2010 to November 2013.

\section{Study selection and risk of bias assessment}

We assessed the appropriateness of each study for meeting the objectives and design of this individual patient data (IPD) meta-analysis. Firstly, we selected studies that documented acute myocardial infarction separately from other cardiovascular outcomes, compared the risk of acute myocardial infarction in NSAID users with non-users, considered traditional and COX 2 selective NSAIDs separately, were conducted in the general or an elderly population, allowed for analyses corresponding to exposure time windows of interest, and minimised the effects of confounding by indication or by contraindication and of selective prescribing. This last criterion was implemented by limiting the inclusion of studies to those that were conducted before rofecoxib was withdrawn from the market, matched cases and controls for calendar time, and documented comorbidities and concomitant treatments. Secondly, we checked that the study was suitable for characterising the time course of myocardial infarction risk and the dose and duration effects of NSAIDs.

\section{Data sources}

The full text of 82 studies was screened for eligibility. Of these, we excluded 67 on the basis of one or more of the criteria outlined previously. Of the 15 studies eligible for further consideration, seven were excluded because the definition of exposed time precluded valid pooling of patient level data, whereas permission to access IPD was not obtained for another four studies. In web appendix 1, figure 1 shows the study flow and tables 2 to 4 the reasons for exclusion of studies.

We created a nested case-control dataset to serve as reference for harmonising the IPD. For the reference dataset (RAMQ) we used data from the universal, computerised public insurance database of Quebec, Canada. The other datasets available for IPD meta-analysis were a population based case-control study from Finland ${ }^{9}$ (Finland) and two nested case-control studies, one from the UK using data from the General Practice Research Database (GPRD) ${ }^{10}$ and the other from Canada using data from Saskatchewan (see table 1 in web appendix 2). ${ }^{11}$ All source databases have been extensively used in pharmacoepidemiology ${ }^{12-15}$ and were validated for the study of coronary heart disease or acute myocardial infarction. ${ }^{16-20}$

\section{Common data structure}

\section{Outcome}

In all studies, the index date was the date of hospital admission with acute myocardial infarction for cases and a time matched date for controls. The similarity of definition and ascertainment of cases and controls in each study see (table 2 in web appendix 2) confirmed the appropriateness of pooling in a meta-analysis.

\section{NSAID exposure and confounders}

The IPD were drawn from the elderly ( $\geq 65$ years) and general populations in the RAMQ and Finland studies, respectively (approximately 200000 individuals each). For the GPRD and Saskatchewan studies the IPD were drawn from the general population (approximately 20000 individuals each). As a preliminary step, we defined a common structure for exposures and confounders to allow for retrospective harmonisation of the data. $^{21}$ The harmonisation framework ensured that studies were clinically and methodologically similar and that patient level data across studies were sufficiently compatible to allow valid data integration. ${ }^{22}$

The NSAIDs of interest were celecoxib, the three main traditional NSAIDs (diclofenac, ibuprofen, and naproxen), and rofecoxib. We captured time varying NSAID use, working with the original study (see table 3 in web appendix 2), to create several multidimensional categories with different indicator variables representing the contribution of recency of use (past, recent, or 

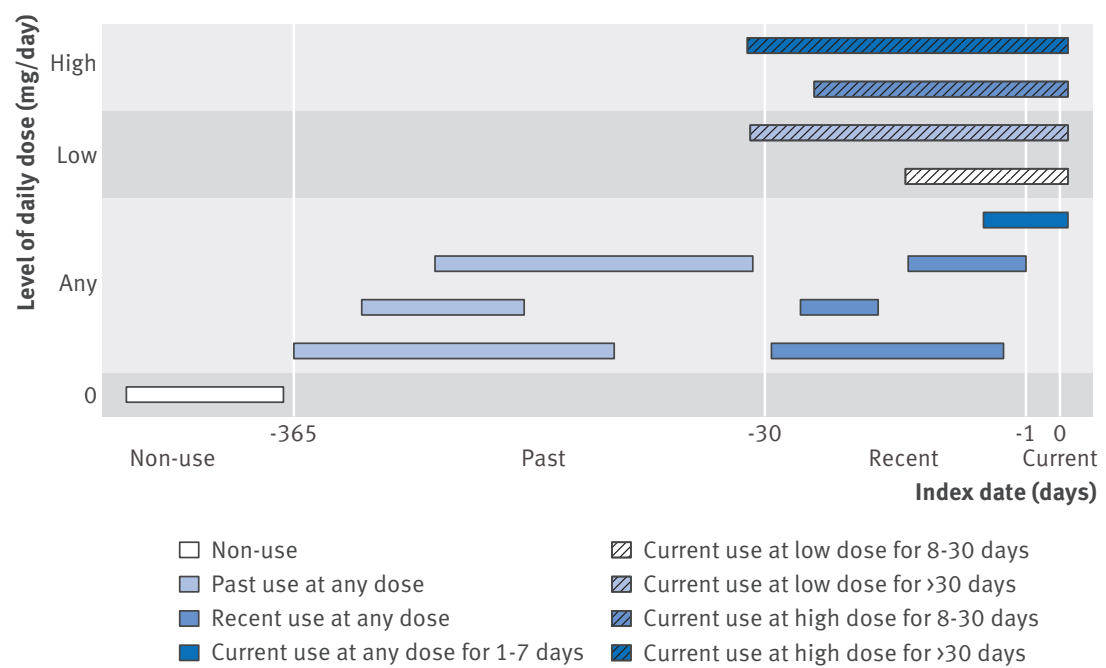

Z7 Current use at low dose for 8-30 days $\square$ Current use at low dose for $>30$ days

$\square$ Current use at high dose for 8-30 days

Current use at high dose for $>30$ days

Fig 1 Multidimensional indicator categories of non-steroidal anti-inflammatory drug (NSAID) use defined by recency of use, daily dose, and duration

current), dose level (low or high), and treatment duration for mutually exclusive time windows (fig 1). We verified that the NSAID use categories in each dataset were inferentially equivalent such that pooling across studies was justified.

On the basis of substantive knowledge and confirmation by a search of the literature, ${ }^{23-26}$ we identified risk factors for the outcome and potential confounders, which formed a set of candidate covariates to include in multivariable regression analysis. Using a simplified causal graph, ${ }^{27}$ we mapped relations between variables, ${ }^{28}$ including the special case of time dependent confounders that are mediating intermediates on the causal pathway between NSAID exposure and the acute myocardial infarction outcome (see fig 1 in web appendix 2). ${ }^{29}$ Such mediator variables must be identified at the planning stage of analysis as they require appropriate measurement and analytical strategies. We then substantiated the confounder status for each candidate covariate by calculating the odds ratio of association between the covariate and exposure to NSAIDs among controls and the odds ratio of association between the covariate and myocardial infarction outcome in the unexposed. This led to the final set of target confounders (or IPD meta-analysis confounders) selected for adjustment in the meta-analysis: age at index date, male sex, diabetes, hyperlipidaemia, hypertension, previous myocardial infarction, coronary heart disease (excluding previous myocardial infarction), congestive heart failure, cerebrovascular disease, peripheral vascular disease, chronic obstructive pulmonary disease, gastrointestinal ulcer disease, gastrointestinal bleed, acute or chronic renal failure, rheumatoid arthritis, and concomitant treatment with oral corticosteroids, clopidogrel, or cardioprotective aspirin. All studies were

adjusted for the following IPD meta-analysis confounders that were common to all studies (or common IPD meta-analysis confounders): age at index date, diabetes, hyperlipidaemia, hypertension, coronary heart disease, and rheumatoid arthritis. Each study was also adjusted for other IPD meta-analysis confounders if available in the original study dataset (see table 2 in web appendix 2). When an IPD meta-analysis confounder was unavailable, it was missing systematically for all subjects in that study.

\section{Statistical analysis}

The available data for the IPD meta-analysis were gathered at two levels. At the higher level of analysis are the characteristics of the four studies. At the lower level are individual patient characteristics. For pooling IPD, we opted for a one stage model in the bayesian framework. Such a model has a hierarchical structure allowing the IPD to be combined in one step while accounting for clustering of data within each database study. ${ }^{30} 31$

In bayesian analysis, combining prior information with the available data yields a posterior probability distribution for each parameter of interest. To avoid undue influence on posterior estimates of non-informative priors for the between study variance, especially since only four studies were available, ${ }^{3233}$ we selected a range of reasonably informative priors based on historical information. ${ }^{3435}$ In a comprehensive recent meta-analysis of observational studies, the odds ratio of acute myocardial infarction with a given NSAID generally varied by up to fourfold across studies and subgroup analyses. ${ }^{36}$ For the primary analysis, the between study standard deviation on the log odds ratio, $\tau$, was assigned a half normal prior distribution (to represent a prior belief that although the true odds ratios of $95 \%$ of studies could span a fourfold range, less heterogeneity is more likely). ${ }^{34}$ For the pooled (log) odds ratio estimates of acute myocardial infarction for each exposure, we used a non-informative normal prior distribution (mean 0, variance $10^{-6}$ ).

A few exposure categories in the Finland and GPRD studies and certain IPD meta-analysis confounders in studies other than from RAMQ were missing systematically. We assumed that it was plausible for systematically missing variables to be missing completely at random. ${ }^{37}$ We specified random effects for each multidimensional indicator category of NSAID use. As appropriate for each study design (RAMQ and GPRD: individually matched nested case-control studies; Finland: population based matched case-control study; Saskatchewan: frequency matched nested case-control study), we implemented conditional (RAMQ, Finland, and GPRD) or unconditional logistic regression (Saskatchewan) to analyse the IPD. For each exposure, we obtained from the posterior distribution the median value of the odds ratio of acute myocardial infarction with its 95\% credible intervals adjusted for the IPD meta-analysis confounders available in each study. We report summary adjusted odds ratios for each exposure category, with non-use of any NSAIDs in the year preceding the index date as the reference category. 
The bayesian framework allows for the making of direct probability statements, based on posterior distributions. For exposures corresponding to current use for each NSAID, we obtained probabilities that the summary adjusted odds ratio of acute myocardial infarction was greater than a series of prespecified thresholds of harms. These probabilities were compiled from the posterior distribution by repeated sampling using a function that took the value of 1 each time the summary adjusted odds ratio exceeded a given odds ratio threshold, and took the value of 0 otherwise. ${ }^{38}$

Bayesian analyses were performed using JAGS ${ }^{39}$ (version 3.4.0) and $\mathrm{R}^{40}$ (version 3.0.3; Foundation for Statistical Computing). All Markov Chain Monte Carlo simulations were run on two chains with 10000 iterations, discarding the first 1000. Convergence was monitored using CODA $^{41}$ (version 0.16-1) and checked by observing the traces of posterior samples, and by inspecting the Gelman-Rubin R statistic ${ }^{42}$ and the Raftery-Louis diagnostics. ${ }^{43}$ We assessed the heterogeneity of effects of NSAID use by examining the medians of between study standard deviation. Web appendix 3 provides additional information on the statistical analysis.

\section{Sensitivity and exploratory analyses Heterogeneity}

In sensitivity analyses for modelling the heterogeneity that may exist between studies, we considered three alternative prior distributions for the between study standard deviation on the log odds ratio, $\tau$. These alternatives were one less informative half normal prior allowing for an eightfold variation in the odds ratios and two uniform priors equally supporting all values of $\tau$ and representing approximately twofold and fourfold variation in odds ratios of acute myocardial infarction across the four studies, respectively.
In exploratory analysis, we compared different drug use categories by calculating the bayesian posterior probability that summary adjusted odds ratios of acute myocardial infarction associated with a given NSAID exceeded those for another NSAID. ${ }^{38}$

\section{Risk of bias}

To examine how imperfect adjustment resulting from systematically missing confounders might affect estimates of acute myocardial infarction, we used two adjustment strategies. We compared results of the RAMQ study obtained with the full set of 18 IPD meta-analysis confounders with only the six common IPD meta-analysis confounders.

\section{Patient involvement}

No patients were involved in setting the research question or the outcome measures, nor were they involved in developing plans for design or implementation of the study. No patients were asked to advise on interpretation or writing up of results. There are no plans to disseminate the results of the research to study participants or the relevant patient community. Our results may be shared through patient and public involvement initiatives established by developers of clinical practice guidelines discussing the use of NSAIDs.

\section{Results}

The pooled data comprised 61460 cases and 385303 controls for a total number of 446763 individuals. Table 1 gives the prevalence of IPD meta-analysis confounders at the index date in each study, which confirms that the populations differed. Despite differences in patient age, the RAMQ and Saskatchewan studies share a high prevalence of hyperlipidaemia and hypertension diagnoses. This suggests that geographical

\begin{tabular}{|c|c|c|c|c|}
\hline Confounders & RAMQ ( $\mathrm{n}=233816)$ & Finland $(n=172219)$ & GPRD $(n=17561)$ & Saskatchewan $(n=23167)$ \\
\hline Mean (SD) age at index date (years) & $77.8(6.1)$ & $68.9(12.7)$ & $70.2(11.5)$ & $58.1(12.8)$ \\
\hline Median (interquartile range) age at index date (years) & $78(73-83)$ & $70(60-78)$ & $71(62-79)$ & $56(47-69)$ \\
\hline Male sex & $118492(50.7)$ & $107225(62.3)$ & $10349(58.9)$ & $11831(51.1)$ \\
\hline \multicolumn{5}{|l|}{ Comorbidities: } \\
\hline Diabetes & $40812(17.5)$ & $12911(7.5)$ & $1933(11.0)$ & $1663(7.2)$ \\
\hline Hyperlipidaemia & $72008(30.8)$ & $19212(11.2)$ & $2397(13.7)$ & $6738(29.1)$ \\
\hline Hypertension & $108916(46.6)$ & $44702(26.0)$ & $5944(33.9)$ & $9181(39.6)$ \\
\hline Previous myocardial infarction & $17025(7.3)$ & NA & NA & $1154(5.0)$ \\
\hline Coronary heart disease & $79466(34.0)$ & $29998(17.4)$ & $3731(21.3)$ & $4972(21.5)$ \\
\hline Congestive heart failure & $19602(8.4)$ & NA & NA & $1722(7.4)$ \\
\hline Cerebrovascular disease & $22203(9.5)$ & NA & $1480(8.4)$ & $1798(7.8)$ \\
\hline Peripheral vascular disease & $15833(6.8)$ & NA & NA & $706(3.1)$ \\
\hline Chronic obstructive pulmonary disease & $53465(22.9)$ & NA & NA & $2546(11.0)$ \\
\hline Gastrointestinal ulcer disease & $68062(29.1)$ & NA & NA & $9419(40.7)$ \\
\hline Gastrointestinal bleed & $5686(2.4)$ & NA & NA & $1039(4.5)$ \\
\hline Acute or chronic renal failure & $4102(1.8)$ & NA & NA & $148(0.6)$ \\
\hline Rheumatoid arthritis & $4245(1.8)$ & $5180(3.0)$ & $574(3.3)$ & $1277(5.5)$ \\
\hline \multicolumn{5}{|l|}{ Concomitant drug treatment: } \\
\hline Oral corticosteroids & $5301(2.3)$ & NA & NA & NA \\
\hline Clopidogrel & $4007(1.7)$ & $172(0.1)$ & NA & NA \\
\hline Cardioprotective aspirin & $53738(23.0)$ & NA & NA & NA \\
\hline
\end{tabular}

$\mathrm{NA}=$ systematically missing in original study. 
variations in medical practice and in population health contributed to observed differences in baseline characteristics between Canadian and European studies. Specific strategies applied in the assessment of comorbidities may also explain some of the observed differences in prevalence between studies from healthcare databases.

Table 2 reports the adjusted odds ratios of acute myocardial infarction for past use, recent use, and the five dose duration categories of current non-steroidal anti-inflammatory drug (NSAID) use compared with non-use of any NSAID in the year before the index date for each study and for the pooled studies. The results suggest that current NSAID use is associated with increased risks of acute myocardial infarction.

The plots on the left in figure 2 present the posterior probability of exceeding certain risk thresholds for each NSAID in each category of current use. The displayed plots help making a conclusion about degrees of harms of greater magnitude associated with current use of each NSAID.

The forest plots on the right in figure 2 present the summary adjusted odds ratios of acute myocardial infarction in the IPD meta-analysis, by drug for each multidimensional exposure category. Odds ratio point estimates of acute myocardial infarction for current use indicate an associated increase in risk of $20 \%$ to $50 \%$ overall, with possible increases of $75 \%$ for ibuprofen and naproxen and more than a 100\% increase for rofecoxib. Credible intervals reveal that uncertainty about the extent of increased risk is greatest for ibuprofen and naproxen.

Table 3 shows the probability of increased risk of myocardial infarction (ie, the probability that the summary adjusted odds ratio of acute myocardial infarction is $>1.0$ ) with current use of a given NSAID, dose level, and duration of use. The probability of increased risk of myocardial infarction associated with use of an NSAID for one to seven days is $92 \%$ to $99 \%$.

\section{Onset of risk}

The IPD meta-analysis suggests that the risk of myocardial infarction associated with NSAID use increases immediately with exposure. This is illustrated by the position of the gold line in the plots in figure 2, which corresponds to the relevant NSAID use for one to seven days.

\section{Dose effect}

The IPD meta-analysis found a relation between increasing NSAID daily dose and risk of acute myocardial infarction. The effect of the higher dose level is illustrated by the higher relative position of the red line (current use at high dose for 8-30 days) or the blue line (current use at high dose for $>30$ days) or both of these lines on graphs in the plots of figure 2 . This dose effect is also read from table 3 (last column), which shows a greater probability of the odds ratios of myocardial infarction being more than $50 \%$ at the higher dose level. With use for 8-30 days (red and brown lines in fig 2), a dose-risk relation is especially noticeable for rofecoxib but is also seen with naproxen and ibuprofen. With use for more than 30 days (blue and lavender lines in fig 2) a dose effect is again observed with rofecoxib and ibuprofen but is no longer distinguishable with naproxen. With diclofenac and celecoxib, more modest dose effects are seen.

\section{Effect of duration of current use}

In the IPD meta-analysis, a longer duration of treatment generally does not seem to be associated with greater probabilities of increased risk of myocardial infarction. Visually, this is indicated by the lower position of the lavender line (current use at low dose for $>30$ days) or the blue line (current use at high dose for $>30$ days) or both of these lines relative to the red, gold, or brown lines ( $\leq 30$ days) in figure 2 . Thus, with the possible exception of diclofenac, there are no obvious further increases in associated risk of myocardial infarction beyond one month compared with use for one month or less.

\section{Recency of use}

The risk of acute myocardial infarction decreases over time since the last use of a NSAID, as shown by odds ratios of acute myocardial infarction for recent use at any dose and past use at any dose categories in figure 2 .

\section{Confidence in magnitude of risk increase associated with short term use}

We examined the plots of bayesian posterior probability that summary adjusted odds ratios of acute myocardial infarction were greater than a series of prespecified thresholds. Selecting a benchmark of $80 \%$ (horizontal black line) and considering use for one to seven days (intersecting gold line), there was at least $80 \%$ probability that the odds ratio of acute myocardial infarction is greater than 1.07 for celecoxib, 1.30 for diclofenac and naproxen, 1.25 for ibuprofen, and 1.35 for rofecoxib (fig 2). Clinicians may wish to consider different cut points for posterior probability of risk and odds ratio thresholds. For example, with $750 \mathrm{mg} /$ day or less of naproxen for 8-30 days, there is a 58\% probability that the odds ratio of acute myocardial infarction is greater than 1.20 (brown line in fig 2).

\section{Heterogeneity of effects}

Values of the between study standard deviations on the log odds ratio summarised across exposure categories had a mean of 0.100 and a median of 0.108 in the primary analysis. For a given category of NSAID use, the odds ratios are similar in magnitude across studies. This indicates good robustness despite populations being heterogeneous and duration of drug use and dose being constructed based on differing sources of raw data. Differences existed in matching strategies and in local prescribing habits during the study period, including uptake of selective COX 2 inhibitors in the various study jurisdictions. ${ }^{44-46}$ These differences may explain the observed contrasting prevalences of exposure on index date within and between studies (table 2). 


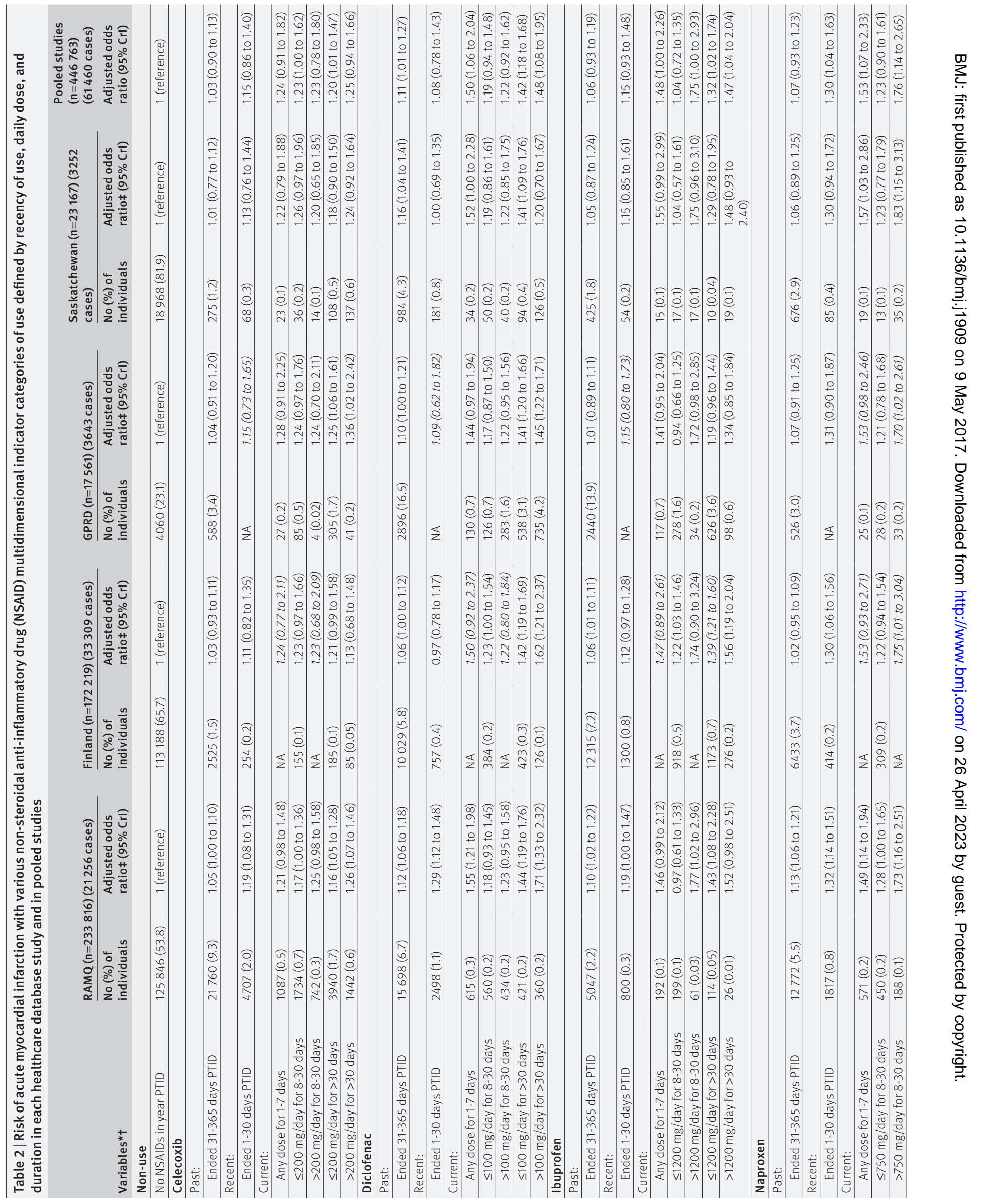




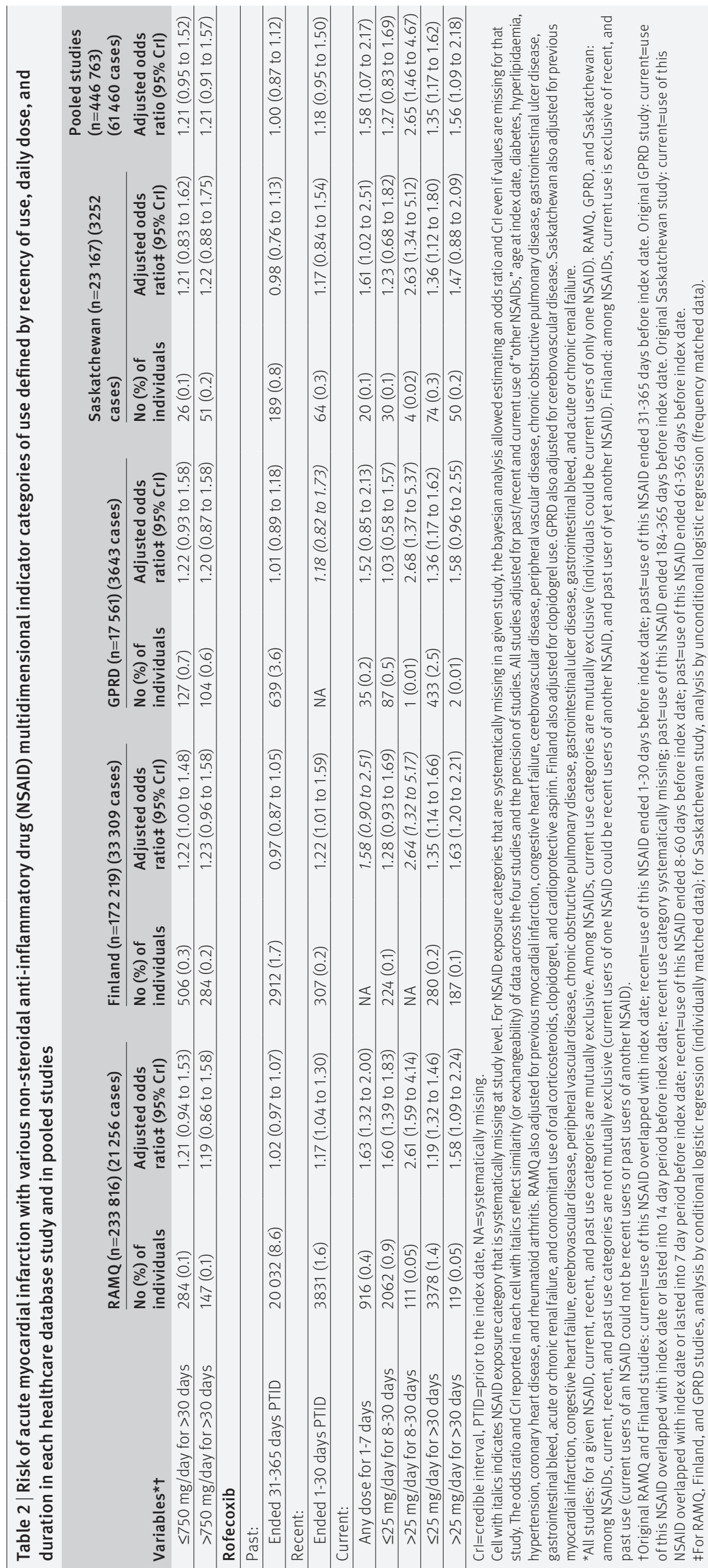

\section{Sensitivity and exploratory analyses}

Table 1 in web appendix 3 reports the results of sensitivity analyses according to selected priors for modelling between study heterogeneity. For a given NSAID category, the posterior median for the summary adjusted odds ratios was relatively consistent across priors. Thus, all four analyses led to similar inferences. Results of model diagnostics indicate that convergence was achieved.

Few consistent patterns emerge in comparisons of NSAIDs based on probability that summary adjusted odds ratios of acute myocardial infarction with a given drug exceeded those for another, except that rofecoxib is associated with more cardiotoxicity than traditional NSAIDs (diclofenac, ibuprofen, and naproxen) and celecoxib (see fig 3 in web appendix 3).

In the sensitivity analysis for imperfect confounding adjustment based on the RAMQ study, only adjusting for the six common IPD meta-analysis confounders had a limited effect on the estimates obtained when adjusting for the full set of 18 IPD meta-analysis confounders (see table 2 in web appendix 3). For exposures and confounders that are completely missing from the IPD meta-analysis common structure, we considered an alternative assumption that these were missing at random and could be obtained by multiple imputation. 4748 We concluded this was implausible in the context of observational studies sourced from healthcare databases (see web appendix 3).

\section{Discussion}

Through its inclusion and exclusion criteria and the definition of exposures, this meta-analysis of individual patient data (IPD) aimed to emulate the design of a large, pragmatic randomised trial ${ }^{49}$ comparing the main non-steroidal anti-inflammatory drugs (NSAIDs) used in the general population. By studying 61460 myocardial infarction events in real world use of NSAIDs, we found that current use of a NSAID is associated with a significantly increased risk of acute myocardial infarction. This was observed for all traditional NSAIDs, including naproxen. In this IPD meta-analysis, the risks of acute myocardial infarction for celecoxib do not appear to be greater than those for traditional NSAIDs and are lower than those for rofecoxib.

NSAIDs exhibited a rapid onset of risk for myocardial infarction in the first week of use. Use for 8-30 days at a high dose was particularly harmful for ibuprofen (>1200 mg/day), naproxen (>750 mg/day), and rofecoxib ( $>25 \mathrm{mg} /$ day) (table 3 and red line in fig 2). The depletion of susceptibles effect $t^{50}$ is a possible explanation for the spikes in risk observed with all NSAIDs. For celecoxib and diclofenac, a single wave of acute myocardial infarction cases occurred within one week (table 3, fig 2). For ibuprofen, naproxen, and rofecoxib, there was additionally a subsequent wave of myocardial infarction cases taking place within 8-30 days of use at a high dose (table 3, fig 2, and fig 4 in web appendix 3). With longer term ( $>30$ days) use of NSAIDs the risk of myocardial infarction may not appear greater for that observation period (except maybe for diclofenac, fig 2). 
P. Past use at any dose

R. Recent use at any dose

- - WK. Current use at any dose for 1-7 days

- - - LS. Current use at low dose for 8-30 days

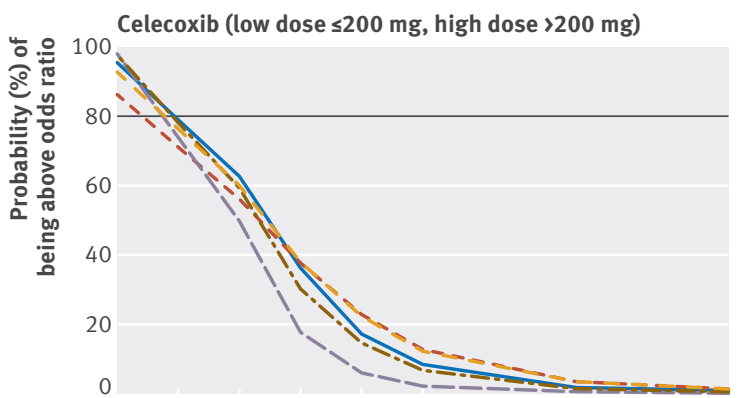

Diclofenac (low dose $\leq 100 \mathrm{mg}$, high dose >100 mg)
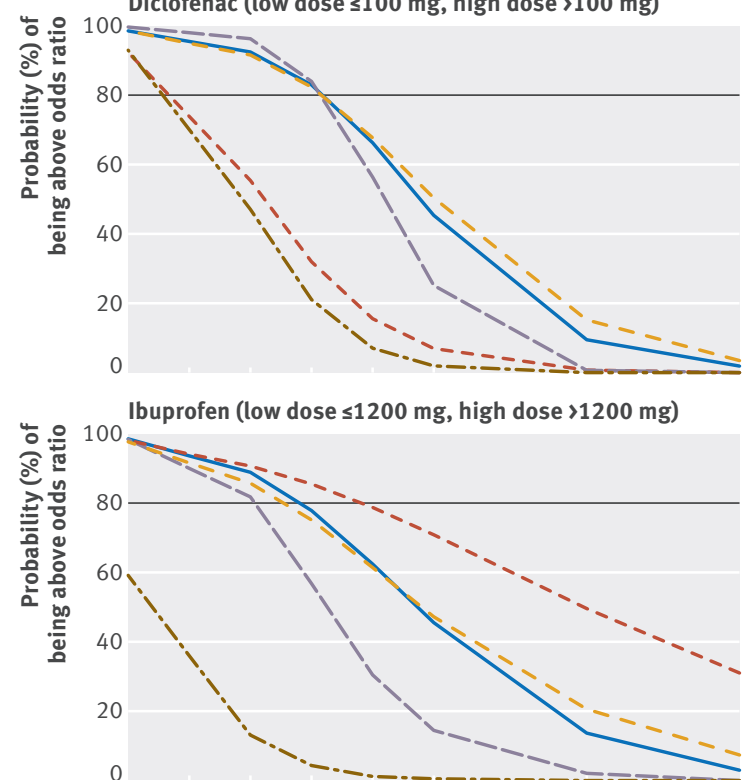

Naproxen (low dose $\leq 750 \mathrm{mg}$, high dose $>750 \mathrm{mg}$ )

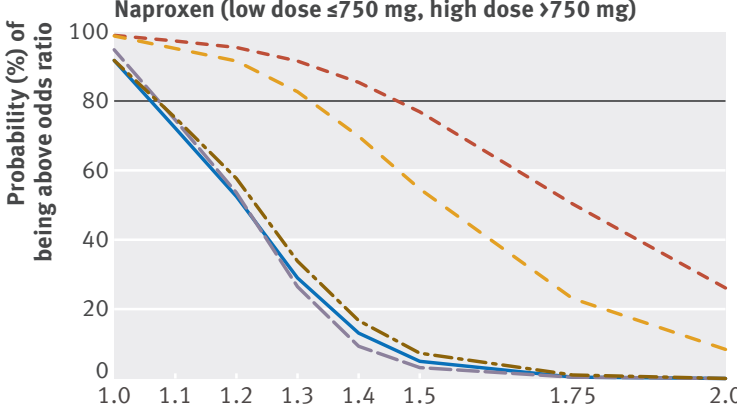

Threshold odds ratio of acute $M$ for current dose duration category

Fig 2 | Plot of probability of exceeding odds ratios of acute myocardial infarction (MI) for exposure categories corresponding to current use for each non-steroidal anti-inflammatory drug (NSAID) versus non-use and corresponding forest plot for risk of acute myocardial infarction for each exposure category in pooled studies

Rofecoxib (low dose $\leq 25 \mathrm{mg}$, high dose $>25 \mathrm{mg}$ )

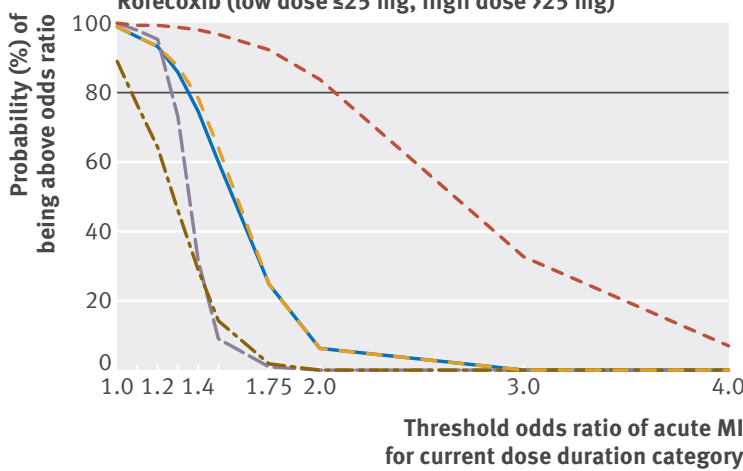

- - - HS. Current use at high dose for 8-30 days

- - LL. Current use at low dose for $>30$ days

- HL. Current use at high dose for $>30$ days
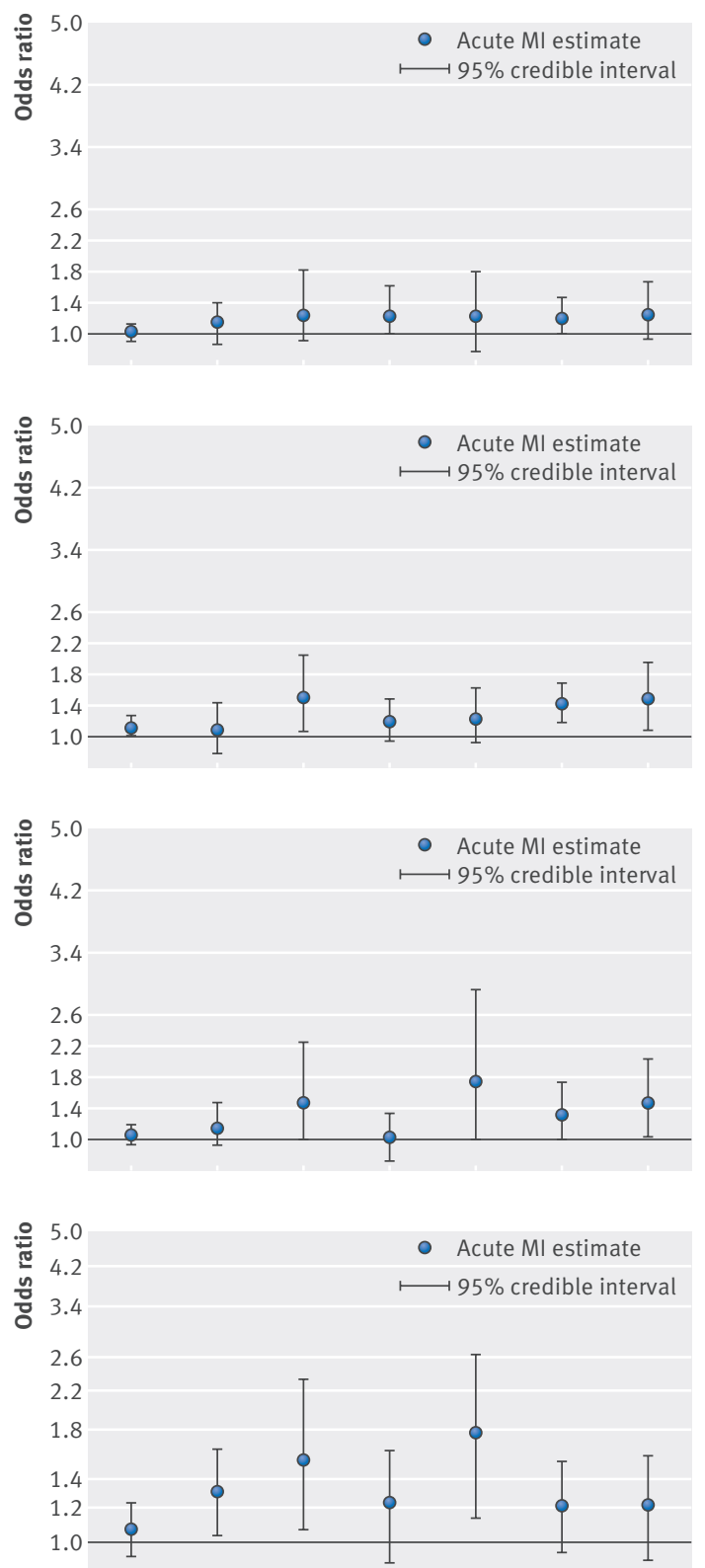


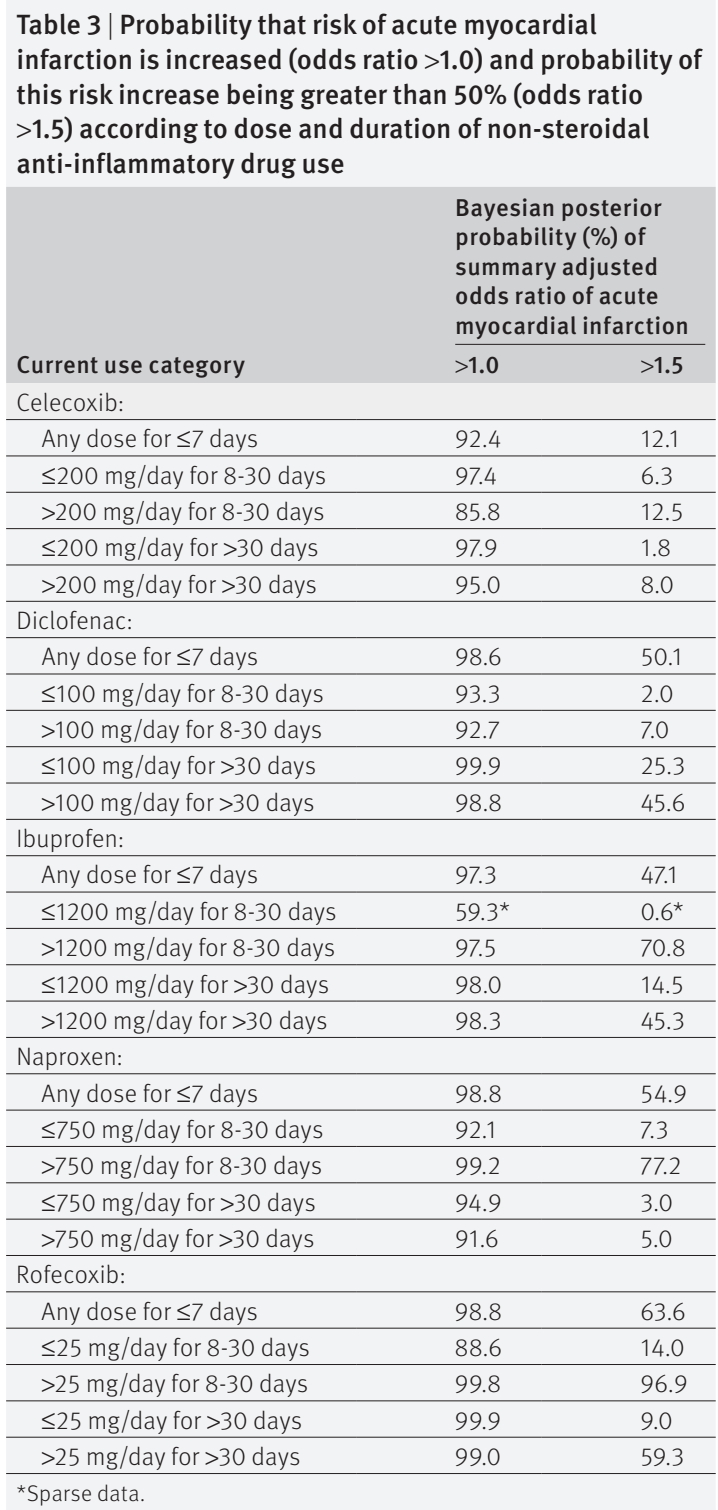

This may be because those more susceptible to acute myocardial infarction were selected out of the cohort at earlier time points. Quantitative differences among NSAIDs in terms of blood pressure increases or deterioration of renal function (such as those reported in the Prospective Randomized Evaluation of Celecoxib Integrated Safety vs. Ibuprofen Or Naproxen (PRECISION) trial), ${ }^{5}$ might contribute to this depletion of susceptibles effect.

Our findings suggest that the risks of myocardial infarction associated with rofecoxib are greater than those associated with other NSAIDs (fig 2). This feature may explain why the myocardial infarction risk of NSAIDs was uncovered first through rofecoxib trials. Our study suggests a smaller risk of myocardial infarction exists with all other NSAIDs.

\section{Strengths and weaknesses of this study}

Patient level meta-analysis has the specific advantages of improving the coherence of exposure definitions, allowing more consistent adjustment for confounding and accounting for variations over time of exposure and confounder status. ${ }^{51}$ Our work, which draws on large population based cohorts created from administrative health or medical databases, is an innovative application of IPD meta-analysis for studying a drug related adverse event. Access to granular data allowed for more comprehensive evaluation of whether or not studies were sufficiently comparable for pooling, which is not possible for aggregate based meta-analysis. ${ }^{52}$ Data harmonisation reduced the bias due to misclassification of NSAID exposure. Through finely restricted time windows after start of treatment, we could discern successive occurrences of depletion of susceptibles effect and document dose effects and the time course of risk for acute myocardial infarction.

The bayesian approach is useful for decision making. Take, for example, the summary odds ratio of acute myocardial infarction of 2.65 (1.46 to 4.67) with rofecoxib $>25 \mathrm{mg} /$ day for 8-30 days versus non-use. With a frequentist confidence interval, which represents uncertainty through repetition of the experience, all odds ratios from 1.46 to 4.67 might seem equally likely. In contrast, the bayesian approach, although resulting in a numerically similar $95 \%$ credible interval, also allows us to calculate that there is an $83 \%$ probability that this odds ratio of acute myocardial infarction is greater than 2.00. Because of the underlying low between study heterogeneity, information was effectively shared by studies (table 2), allowing more definitive conclusions to be reached.

The common practice in aggregate data meta-analysis is to pool studies irrespective of internal validity, and perhaps to conduct sensitivity analyses based on quality. We opted to assess whether or not candidate studies were adequate at the IPD meta-analysis design stage. This offered important gains in efficiency, improved the control of confounding, and minimised measurement error. Indeed, given the objectives of this meta-analysis, not excluding studies with misclassified exposure time or with incompatible exposure definitions might have induced information bias. Figures 2 to 6 in web appendix 1, created after study selection, suggest this approach was an acceptable trade-off and did not lead to biased selection of studies.

Of eight eligible studies, four ultimately had to be excluded because of ethicolegal restrictions placed by health authorities on transfer of IPD to third parties. Selection bias, denoting a common effect of two variables, ${ }^{53}$ was not expected to occur since denied access to patient level data was completely independent of findings of acute myocardial infarction in the study. Figs 2 to 6 in web appendix 1 show that the unavailability of four studies did not result in bias. However, unavailability did reduce statistical power, affecting the precision around estimates of myocardial infarction risk in the IPD meta-analysis.

This work shares the limitations of all studies sourced from databases, which measure drug dispensing or drug prescribing and not actual drug intake. In particular, the ibuprofen estimates may be globally more fragile due to the universal availability of over-the-counter 
purchase and because use "as needed" in our data was documented to be more prevalent for ibuprofen than for the other NSAIDs.

Previous papers ${ }^{555}$ provide useful insight on the risk of bias due to confounders unobserved in studies from databases (obesity, over-the-counter aspirin or NSAID use, smoking, income, or educational attainment), which suggests that failure to adjust for these confounders might slightly underestimate the risk of myocardial infarction. ${ }^{56}$ We suspect that residual confounding exists because substantive knowledge ${ }^{57-59}$ ascertains that there are mediating intermediate variables on the causal pathway between NSAID exposures and acute myocardial infarction (see fig 1 in web appendix 2). Analyses of the PRECISION trial ${ }^{5}$ suggest that the main sources of bias from residual confounding in our study are the mediating effects of blood pressure increases or renal deterioration. The likelihood of bias may increase with longer durations of NSAID use over follow-up time, such that the odds ratios of acute myocardial infarction in the exposure categories corresponding to use for longer than 30 days might be biased to the null (underestimated). On the basis of our assessment of the literature 232660 and the anticipated direction of bias to the null, we believe that unmeasured and incompletely measured confounders are unlikely to affect the substantive conclusions of this IPD meta-analysis, which found associations between current NSAID use and increased risk of acute myocardial infarction.

\section{Comparison with other studies}

The patients enrolled in the PRECISION randomised controlled trial required regular daily NSAID treatment for arthritis and received rather high doses of ibuprofen (2045 (SD 246) mg) and naproxen (852 (103) mg). Permitted doses of celecoxib (209 (37) $\mathrm{mg}$ ) were at the low-moderate end of the therapeutic range. The results of this IPD meta-analysis of studies from healthcare databases are aligned with the main conclusions of the PRECISION trial, which concluded that in studied dosages, celecoxib was non-inferior to ibuprofen or naproxen for cardiovascular safety. ${ }^{5}$ Similar to our observational findings, the PRECISION trial does not provide evidence to suggest that naproxen is safer than other NSAIDs.

Summaries of risk for acute myocardial infarction with NSAIDs obtained in placebo controlled trials are available from two network meta-analyses, one with aggregate data ${ }^{4}$ and the other with IPD. ${ }^{3}$ The bulk of placebo controlled direct evidence suggesting a neutral effect for naproxen came from randomised controlled trials in populations with, or at risk of, Alzheimer's disease. ${ }^{4}$ Non-adherence bias, documented in such patient populations, ${ }^{61}$ may translate into an underestimation of the risk with naproxen in meta-analyses of randomised controlled trials. The risk of myocardial infarction was not considered separately for the various selective COX 2 inhibitors in the IPD network meta-analysis of randomised controlled trials. ${ }^{3}$ Our results indicate that there seem to be differences between rofecoxib and celecoxib on risk of acute myocardial infarction, which agrees with the findings for celecoxib in the PRECISION trial. ${ }^{5}$

Estimates of risks for acute myocardial infarction found by the Safety of Non-Steroidal Anti-Inflammatory (SOS) Project meta-analysis of observational studies $^{36}$ are overall lower than those of our IPD meta-analysis. In accordance with most aggregate meta-analyses, no restriction based on methodological quality was imposed in the SOS meta-analysis such that it included field case-control studies possibly affected by recall bias, ${ }^{62} 63$ a cohort study with immortal time bias, ${ }^{64}$ studies that compared risk in current users with users in the recent past, ${ }^{5465}$ and studies defining as current use a time window possibly remote from the event date. ${ }^{66} 67$ These dissimilarities and potential biases, if occurring in a non-differential manner, would be expected to move pooled odds ratio of acute myocardial infarction towards the null (see figs 2 to 6 in web appendix 1).

In the Adenomatous Polyp Prevention on Vioxx (APPROVe) trial, the largest individual trial of rofecoxib (25 mg) versus placebo, the hazard ratio of acute myocardial infarction up to 14 days after end of treatment was 2.65 (95\% confidence interval 1.21 to 5.75$).{ }^{68}$ The Trelle network meta-analysis of randomised controlled trials reported a rate ratio for rofecoxib (any daily dose) versus placebo of 2.12 (95\% credible interval 1.26 to 3.56). ${ }^{4}$ A weighted average of the IPD meta-analysis findings across the five categories of current rofecoxib use (see table 2 and fig 2) would yield a lower risk than that of the APPROVe trial and the Trelle network meta-analysis of randomised controlled trials.

\section{Unanswered questions and future research}

We might have taken advantage of this study to investigate clinical heterogeneity using a formal analysis of interactions between NSAIDs and major cardiovascular risk profiles. ${ }^{69-71}$ However, interaction studies involving a categorical NSAID exposure, such as in this IPD meta-analysis, would have less statistical power ${ }^{72}$ and would overly depend on classifying drug exposure without error. ${ }^{73}$

Since methods exist for pooling IPD and aggregate data ${ }^{51}$ we considered performing a combined IPD aggregate data meta-analysis. This proved infeasible because the aggregate data did not permit reconstruction of NSAID exposure as multidimensional categorical variables or adjustment for time dependence of confounders. In our experience, a particular and possibly underappreciated hurdle of IPD meta-analysis sourced from healthcare databases is that they critically depend on health authorities granting permission to access IPD. This lack of willingness to share even deidentified observational data is in direct contrast to the current movement to encourage data sharing of randomised controlled trials ${ }^{74}$ and is a potential barrier to improving comparative effectiveness research.

Even a set of exposure indicators combining recency of use, dose, and duration of treatment, may not suffice to precisely describe the joint impact on myocardial infarction of all components of exposure we assessed in 
this IPD meta-analysis. In particular, we could not study whether the effect of past doses of NSAIDs persisted and affected current risk nor could we determine the precise onset of any associated increased risk or the exact duration of any persistence of risk after stopping an NSAID. In particular, for diclofenac, the risk of myocardial infarction with treatment for more than 30 days (blue and lavender lines in fig 2 and table 3 ) is higher than with treatment for 8-30 days (brown and red lines in fig 2), which hints at cumulative effects for this drug. A more sensitive modelling approach might be needed to finely ascribe risk-for example, after switching between NSAIDs. A recency weighted cumulative exposure model ${ }^{7576}$ might help answer these additional questions.

\section{Conclusions and policy implications}

This patient level meta-analysis of acute myocardial infarction associated with NSAIDs involving 61460 cases in 446763 individuals is the largest investigation of its type, and its real world origin helps ensure that findings are broadly generalisable. The key advantages of this IPD meta-analysis are its pertinence of populations, power owing to sample size, relevance of exposure measurement, and posterior probability distributions that directly inform clinical practice. That we were able to draw conclusions on the risk of myocardial infarction of NSAID dose levels and treatment durations corresponding to various scenarios of actual use is a novel contribution.

In summary, compared with non-use of NSAIDs in the preceding year, we documented that current use of all studied NSAIDs, including naproxen, was associated with an increased risk of acute myocardial infarction. The risk of myocardial infarction with celecoxib was not found to exceed that of other NSAIDs and was lower than for rofecoxib. Given that the onset of risk of acute myocardial infarction occurred in the first week and appeared greatest in the first month of treatment with higher doses, prescribers should consider weighing the risks and benefits of NSAIDs before instituting treatment, particularly for higher doses.

We thank Mary Rose Stang, research epidemiologist, Population Health Branch, Saskatchewan Ministry of Health, Regina, Canada, for her help. This study is based in part on deidentified data provided by the Saskatchewan Ministry of Health. The interpretation and conclusions contained herein do not necessarily represent those of the Government of Saskatchewan or the Saskatchewan Ministry of Health

Contributors: MB designed the study, prepared the analytical plan, created datasets, performed all statistical analyses, interpreted results, and wrote the first draft of the article. JMB had the original idea, organised collaboration between investigators, and acquired or organised collection of the data. ND and JMB advised on the design and analysis of the study and on interpretation of results. BR conceived the analytical model. LN programmed complex routines and verified locally available datasets for quality assurance. AHS and EG facilitated access to patient level data and provided tools needed for replicating original study results. All authors critically revised the manuscript and contributed to the final draft. MB is the guarantor.

Funding: This study is part of MB's doctoral research thesis in epidemiology. MB received grants from the McGill University Health Centre Research Institute during the conduct of the study.

Competing interests: All authors have completed the ICMJE uniform disclosure form at www.icmje.org/coi_disclosure.pdf and declare: MB reports grants from McGill University Health Centre Research Institute during the conduct of the study and personal fees from Institut national d'excellence en santé et services sociaux, outside the submitted work; JMB reports serving on the Data Monitoring Committee for the PRECISION trial, which is sponsored by Pfizer during the conduct of the study; BR reports personal fees from Certara, outside the submitted work; EG reports personal fees from Astellas, grants and personal fees from Bayer, GSK, Novartis, Takeda, and Nycomed, and grants from STADA, Sanofi-Aventis, and Celgene, outside the submitted work.

Ethical approval: The study was approved by the McGill University Health Centre research ethics board (13-380-SDR).

Data sharing: No additional data are available.

Transparency: The lead author (MB) affirms that the manuscript is an honest, accurate, and transparent account of the study being reported; that no important aspects of the study have been omitted; and that any discrepancies from the study as planned have been explained.

This is an Open Access article distributed in accordance with the Creative Commons Attribution Non Commercial (CC BY-NC 4.0) license, which permits others to distribute, remix, adapt, build upon this work non-commercially, and license their derivative works on different terms, provided the original work is properly cited and the use is non-commercial. See: http://creativecommons.org/licenses/ by-nc/4.0/.

Eichler HG, Abadie E, Breckenridge A, et al. Bridging the efficacy-effectiveness gap: a regulator's perspective on addressing variability of drug response. Nat Rev Drug Discov 2011;10:495-506. doi:10.1038/nrd3501.

2 Rothwell PM. Factors that can affect the external validity of randomised controlled trials. PLoS Clin Trials 2006;1:e9. doi:10.1371/ journal.pctr.0010009.

3 Coxib and traditional NSAID Trialists' (CNT) CollaborationBhala N, Emberson J, Merhi A, et al. Vascular and upper gastrointestinal effects of non-steroidal anti-inflammatory drugs: meta-analyses of individual participant data from randomised trials. Lancet 2013;382:769-79. doi:10.1016/S0140-6736(13)60900-9.

4 Trelle S, Reichenbach S, Wandel S, et al. Cardiovascular safety of non-steroidal anti-inflammatory drugs: network meta-analysis. BM 2011;342:c7086. doi:10.1136/bmj.c7086

5 Nissen SE, Yeomans ND, Solomon DH, et al. PRECISION Trial Investigators. Cardiovascular Safety of Celecoxib, Naproxen, or Ibuprofen for Arthritis. N Engl J Med 2016;375:2519-29. doi:10.1056/ NEJMoa1611593.

6 Gore M, Sadosky A, Leslie D, Tai KS, Seleznick M. Patterns of therapy switching, augmentation, and discontinuation after initiation of treatment with select medications in patients with osteoarthritis. Clin Ther 2011;33:1914-31. doi:10.1016/j.clinthera.2011.10.019.

7 Langman M, Kahler KH, Kong SX, et al. Drug switching patterns among patients taking non-steroidal anti-inflammatory drugs: a retrospective cohort study of a general practitioners database in the United Kingdom. Pharmacoepidemiol Drug Saf 2001;10:517-24. doi:10.1002/pds.653.

8 Sift R, van Staa TP, Abenhaim L, Ebner D. A study of the longitudinal utilization and switching-patterns of non-steroidal anti-inflammatory drugs using a pharmacy based approach. Pharmacoepidemiol Drug Saf 1997;6:263-8. doi:10.1002/

(SICI)1099-1557(199707)6:4<263::AID-PDS286>3.0.CO;2-Z

9 Helin-Salmivaara A, Virtanen A, Vesalainen R, et al. NSAID use and the risk of hospitalization for first myocardial infarction in the general population: a nationwide case-control study from Finland. Eur Heart J 2006;27:1657-63. doi:10.1093/eurheartj/ehl053.

10 Andersohn F, Suissa S, Garbe E. Use of first- and second-generation cyclooxygenase-2-selective nonsteroidal antiinflammatory drugs and risk of acute myocardial infarction. Circulation 2006;113:1950-7. doi:10.1161/CIRCULATIONAHA.105.602425.

11 Varas-Lorenzo C, Castellsague J, Stang MR, Perez-Gutthann S, Aguado J, Rodriguez LA. The use of selective cyclooxygenase-2 inhibitors and the risk of acute myocardial infarction in Saskatchewan, Canada. Pharmacoepidemiol Drug Saf 2009;18:1016-25. doi:10.1002/ pds.1815.

12 Herrett E, Thomas SL, Schoonen WM, Smeeth L, Hall AJ. Validation and validity of diagnoses in the General Practice Research Database: a systematic review. Br J Clin Pharmacol 2010;69:4-14. doi:10.1111/j.1365-2125.2009.03537.x

13 Rawson NS, Downey W, Maxwell CI, West R. 25 years of pharmacoepidemiologic innovation: the Saskatchewan health administrative databases. J Popul Ther Clin Pharmacol 2011;18:e245-9.

14 Tamblyn R, Lavoie G, Petrella L, Monette J. The use of prescription claims databases in pharmacoepidemiological research: the accuracy and comprehensiveness of the prescription claims database in Québec. / Clin Epidemiol 1995; 48:999-1009.

doi:10.1016/0895-4356(94)00234-H. 
15 Wettermark B, Zoëga H, Furu K, et al. The Nordic prescription databases as a resource for pharmacoepidemiological research--a literature review. Pharmacoepidemiol Drug Saf 2013;22:691-9. doi:10.1002/pds.3457

16 Hammad TA, McAdams MA, Feight A, lyasu S, Dal Pan GJ. Determining the predictive value of Read/OXMIS codes to identify incident acute myocardial infarction in the General Practice Research Database. Pharmacoepidemiol Drug Saf 2008;17:1197-201. doi:10.1002/ pds.1672

17 Lambert L, Blais C, Hamel D, et al. Evaluation of care and surveillance of cardiovascular disease: can we trust medico-administrative hospital data?Can J Cardiol 2012;28:162-8. doi:10.1016/j. cjca.2011.10.005.

18 Levy AR, Tamblyn RM, Fitchett D, McLeod PJ, Hanley JA. Coding accuracy of hospital discharge data for elderly survivors of myocardial infarction. Can / Cardiol 1999:15:1277-82

19 Pajunen P, Koukkunen H, Ketonen M, et al. The validity of the Finnish Hospital Discharge Register and Causes of Death Register data on coronary heart disease. Eur J Cardiovasc Prev Rehabil 2005;12:132-7.

20 Varas-Lorenzo C, Castellsague J, Stang MR, Tomas L, Aguado J. Perez-Gutthann S. Positive predictive value of ICD-9 codes 410 and 411 in the identification of cases of acute coronary syndromes in the Saskatchewan Hospital automated database. Pharmacoepidemiol Drug Saf 2008;17:842-52. doi:10.1002/pds.1619.

21 Fortier I, Doiron D, Little J, et al. International Harmonization Initiative. Is rigorous retrospective harmonization possible? Application of the DataSHaPER approach across 53 large studies. Int J Epidemio 2011;40:1314-28. doi:10.1093/ije/dyr106.

22 Fortier I, Doiron D, Burton P, Raina P. Invited commentary: consolidating data harmonization--how to obtain quality and applicability?Am J Epidemiol 2011;174:261-4, author reply 265-6 doi:10.1093/aje/kwr194

23 Coca SG, Yusuf B, Shlipak MG, Garg AX, Parikh CR. Long-term risk of mortality and other adverse outcomes after acute kidney injury: a systematic review and meta-analysis. Am J Kidney Dis 2009;53:96173. doi:10.1053/j.ajkd.2008.11.034

24 Hall AJ, Stubbs B, Mamas MA, et al. Association between osteoarthritis and cardiovascular disease: systematic review and meta-analysis. Eur J Prev Cardiol 2016;23:938-46.

25 Han MK, McLaughlin VV, Criner GJ, Martinez FJ. Pulmonary diseases and the heart. Circulation 2007:116:2992-3005. doi:10.1161/ CIRCULATIONAHA.106.685206.

26 Meisinger C, Döring A, Löwel H. KORA Study Group. Chronic kidney disease and risk of incident myocardial infarction and all-cause and cardiovascular disease mortality in middle-aged men and women from the general population. Eur Heart J 2006;27:1245-50. doi:10.1093/eurheartj/ehi880.

27 Greenland S, Pearl J, Robins JM. Causal diagrams for epidemiologic research. Epidemiology 1999;10:37-48 doi:10.1097/00001648-199901000-00008

28 Sauer BC, Brookhart MA, Roy J, VanderWeele T. A review of covariate selection for non-experimental comparative effectiveness research. Pharmacoepidemiol Drug Saf2013;22:1139-45. doi:10.1002/pds.3506.

29 Robins JM, Hernán MA, Brumback B. Marginal structural models and causal inference in epidemiology. Epidemiology 2000;11:550-60. doi:10.1097/00001648-200009000-00011.

30 Debray TP, Moons KG, Abo-Zaid GM, Koffijberg H, Riley RD. Individual participant data meta-analysis for a binary outcome: one-stage or two-stage?PLoS One 2013;8:e60650. doi:10.1371/journal. pone.0060650.

31 Stewart GB, Altman DG, Askie LM, Duley L, Simmonds MC, Stewart LA. Statistical analysis of individual participant data meta-analyses: a comparison of methods and recommendations for practice. PLoS One 2012;7:e46042. doi:10.1371/journal.pone.0046042.

32 Daniels MJ. A prior for the variance in hierarchical models. Can J Stat 1999;27:567-78doi:10.2307/3316112.

33 Lambert PC, Sutton AJ, Burton PR, Abrams KR, Jones DR. How vague is vague? A simulation study of the impact of the use of vague prio distributions in MCMC using WinBUGS. Stat Med 2005;24:2401-28. doi:10.1002/sim.2112.

34 Spiegelhalter DJ, Abrams KR, Myles JP. Prior Distributions. Bayesian Approaches to Clinical Trials and Health-Care Evaluation.John Wiley \& Sons, Ltd, 2004: 139-80doi:10.1002/0470092602.ch5.

35 Higgins JP, Whitehead A. Borrowing strength from external trials in a meta-analysis. Stat Med 1996:15:2733-49. doi:10.1002/ (SICI)1097-0258(19961230)15:24<2733::AID-SIM562>3.0.CO;2-0

36 Varas-Lorenzo C, Riera-Guardia N, Calingaert B, et al. Myocardial infarction and individual nonsteroidal anti-inflammatory drugs meta-analysis of observational studies. Pharmacoepidemiol Drug Saf 2013;22:559-70. doi:10.1002/pds.3437.

37 Little RJA, Rubin DB. Statistical Analysis with Missing Data (2nd Edition). Part 1. Overview and basic approaches. Somerset NJ, USA. 2002.

38 Welton NJ, Sutton AJ, Cooper N, et al. Bayesian methods and WinBUGS. In: Evidence Synthesis for Decision Making in Healthcare.John Wiley \& Sons Ltd, 2012: 17-42doi:10.1002/9781119942986.ch2.
39 Plummer M, Stukalov A. Package ‘rjags'. Bayesian graphical models using MCMC. October 27, 2014. Available at: https://cran.r-project. org/web/packages/rjags/rjags.pdf.

40 R Core Team. R: A Language and Environment for Statistical Computing. Vienna, Austria: R Foundation for Statistical Computing. 2014. Retrieved from http://www.R-project.org/.

41 Plummer M, Best N, Cowles K, et al. Package 'coda'. Output analysis and diagnostics for Markov Chain Monte Carlo simulations. July 2, 2014. Available at: https://cran.r-project.org/web/packages/coda/ coda.pdf.

42 Gelman A, Rubin DB. Inference from iterative simulation using multiple sequences. Stat Sci 1992;7:457-511doi:10.1214/ ss/1177011136.

43 Raftery AE, Lewis SM. One long run with diagnostics: Implementation strategies for Markov chain Monte Carlo. Stat Sci 1992;7:4937doi:10.1214/ss/1177011143.

44 Girvin B, Rafferty T, Stevenson MR, Johnston GD. Uptake of COX-2 selective inhibitors and influence on NSAID prescribing in Northern Ireland. Pharmacoepidemiol Drug Saf 2004;13:153-7. doi:10.1002/ pds.866.

45 Helin-Salmivaara A, Huupponen R, Virtanen A, Klaukka T. Adoption of celecoxib and rofecoxib: a nationwide database study. I Clin Pharm Ther 2005;30:145-52. doi:10.1111/j.1365-2710.2005.00627.x.

46 Mamdani M, Rochon P, Laupacis A, Anderson G. Initial patterns of use of COX-2 inhibitors by elderly patients in Ontario: findings and implications. CMA/ 2002;167:1125-6.

47 Jolani S, Debray TPA, Koffijberg H, van Buuren S, Moons KG. Imputation of systematically missing predictors in an individual participant data meta-analysis: a generalized approach using MICE. Stat Med 2015;34:1841-63. doi:10.1002/sim.6451.

48 Resche-Rigon M, White IR, Bartlett JW, Peters SA, Thompson SG. PROG-IMT Study Group. Multiple imputation for handling systematically missing confounders in meta-analysis of individual participant data. Stat Med 2013;32:4890-905. doi:10.1002/ sim.5894.

49 Hernán MA, Robins JM. Using Big Data to emulate a target trial when a randomized trial is not available. Am J Epidemiol 2016;183:758-64. doi:10.1093/aje/kwv254.

50 Moride Y, Abenhaim L. Evidence of the depletion of susceptibles effect in non-experimental pharmacoepidemiologic research. J Clin Epidemiol 1994:47:731-7. doi:10.1016/0895-4356(94)90170-8.

51 Debray TP, Moons KG, van Valkenhoef G, et al. GetReal Methods Review Group. Get real in individual participant data (IPD) meta-analysis: a review of the methodology. Res Synth Methods 2015;6:293-309. doi:10.1002/jirsm.1160.

52 Warren FC, Abrams KR, Golder S, Sutton AJ. Systematic review of methods used in meta-analyses where a primary outcome is an adverse or unintended event. BMC Med Res Methodol 2012;12:64. doi:10.1186/1471-2288-12-64

53 Hernán MA, Hernández-Díaz S, Robins JM. A structural approach to selection bias. Epidemiology 2004:15:615-25. doi:10.1097/01. ede.0000135174.63482.43.

54 Graham DJ, Campen D, Hui R, et al. Risk of acute myocardial infarction and sudden cardiac death in patients treated with cyclo-oxygenase 2 selective and non-selective non-steroidal anti-inflammatory drugs: nested case-control study. Lancet 2005;365:475-81. doi:10.1016/ S0140-6736(05)70270-1.

55 Solomon DH, Schneeweiss S, Glynn RI, et al Relationship between selective cyclooxygenase-2 inhibitors and acute myocardial infarction in older adults. Circulation 2004;109:2068-73. doi:10.1161/01. CIR.0000127578.21885.3E

56 Schneeweiss S, Glynn RJ, Tsai EH, Avorn J, Solomon DH. Adjusting for unmeasured confounders in pharmacoepidemiologic claims data using external information: the example of $\mathrm{COX} 2$ inhibitors and myocardial infarction. Epidemiology 2005;16:17-24. doi:10.1097/01. ede.0000147164.11879.b5

57 Chan CC, Reid CM, Aw TJ, Liew D, Haas SJ, Krum H. Do COX-2 inhibitors raise blood pressure more than nonselective NSAIDs and placebo? An updated meta-analysis. J Hypertens 2009;27:2332-41. doi:10.1097/ HJH.Ob013e3283310dc9.

58 Johnson AG, Nguyen TV, Day RO. Do nonsteroidal anti-inflammatory drugs affect blood pressure? A meta-analysis. Ann Intern Med 1994;121:289-300 doi:10.7326/0003-4819-121-4-199408150-00011

59 Ungprasert P, Cheungpasitporn W, Crowson CS, Matteson EL. Individual non-steroidal anti-inflammatory drugs and risk of acute kidney injury: A systematic review and meta-analysis of observational studies. Eur J Intern Med 2015;26:285-91. doi:10.1016/j. ejim.2015.03.008

60 Collins R, Peto R, MacMahon S, et al. Blood pressure, stroke, and coronary heart disease. Part 2, Short-term reductions in blood pressure: overview of randomised drug trials in their epidemiological context. Lancet 1990;335:827-38. doi:10.1016/0140-6736(90)90944-Z. 
61 Steering Committee of the Alzheimer's Disease Anti-inflammatory Prevention Trial (ADAPT). Statement for communication to the FDA Arthritis Advisory Committee and the Drug Safety and Risk Management Advisory Committee. 18 February 2005. Available at: https://jhuccs1.us/adapt/pdf\%20documents/FDA\%20ADAPT\%20 STATEMENT web\%20posting.pdf. Last accessed December 13, 2015.

62 Bueno H, Bardají A, Patrignani P, Martín-Merino E, García-Rodríguez LA. Spanish Case-Control Study to Assess NSAID-Associated ACS Risk Investigators. Use of non-steroidal antiinflammatory drugs and type-specific risk of acute coronary syndrome. Am J Cardiol 2010;105:1102-6. doi:10.1016/j.amjcard.2009.12.008

63 Kimmel SE, Berlin JA, Reilly M, et al. Patients exposed to rofecoxib and celecoxib have different odds of nonfatal myocardial infarction. Ann Intern Med 2005;142:157-64

doi:10.7326/0003-4819-142-3-200502010-00005.

64 Mamdani M, Rochon P, Juurlink DN, et al. Effect of selective cyclooxygenase 2 inhibitors and naproxen on short-term risk of acute myocardial infarction in the elderly. Arch Intern Med 2003;163:481-6. doi:10.1001/archinte.163.4.481

65 van der Linden MW, van der Bii S, Welsing P, Kuipers EJ, Herings RM. The balance between severe cardiovascular and gastrointestinal events among users of selective and non-selective non-steroidal anti-inflammatory drugs. Ann Rheum Dis 2009;68:668-73. doi:10.1136/ard.2007.087254

66 Abraham NS, El-Serag HB, Hartman C, Richardson P, Deswal A. Cyclooxygenase-2 selectivity of non-steroidal anti-inflammatory drugs and the risk of myocardial infarction and cerebrovascular accident. Aliment Pharmacol Ther 2007;25:913-24 doi:10.1111/j.1365-2036.2007.03292.x.

67 Hippisley-CoxJ, Coupland C. Risk of myocardial infarction in patients taking cyclo-oxygenase-2 inhibitors or conventional non-steroidal anti-inflammatory drugs: population based nested case-control analysis. BM/ 2005;330:1366. doi:10.1136/bmj.330.7504.1366.
68 Baron JA, Sandler RS, Bresalier RS, et al. Cardiovascular events associated with rofecoxib: final analysis of the APPROVe trial. Lancet 2008:372:1756-64. doi:10.1016/S0140-6736(08)61490-7.

69 Debray TP, Moons KG, van Valkenhoef G, et al. GetReal Methods Review Group. Get real in individual participant data (IPD) meta-analysis: a review of the methodology. Res Synth Methods 2015;6:293-309. doi:10.1002/jrsm.1160.

70 Fisher DJ, Copas AJ, Tierney JF, Parmar MK. A critical review of methods for the assessment of patient-level interactions in individual participant data meta-analysis of randomized trials, and guidance for practitioners. J Clin Epidemiol 2011;64:949-67. doi:10.1016/j. jclinepi.2010.11.016.

71 Simmonds MC, Higgins JP. Covariate heterogeneity in meta-analysis: criteria for deciding between meta-regression and individual patient data. Stat Med 2007;26:2982-99. doi:10.1002/sim.2768.

72 Greenland S. Basic problems in interaction assessment. Environ Health Perspect 1993;101(Suppl 4):59-66. doi:10.1289/ ehp.93101s459.

73 Greenland S. Interactions in epidemiology: relevance, identification, and estimation. Epidemiology 2009:20:14-7. doi:10.1097| EDE.0b013e318193e7b5

74 Taichman DB, Backus J, Baethge C, et al. Sharing clinical trial data: a proposal from the International Committee of Medical Journal Editors. Lancet 2016:387:e9-11. doi:10.1016/S0140-6736(15)01279-9.

75 Abrahamowicz M, Bartlett G, Tamblyn R, du Berger R. Modeling cumulative dose and exposure duration provided insights regarding the associations between benzodiazepines and injuries. / Clin Epidemiol 2006;59:393-403. doi:10.1016/j.jclinepi.2005.01.021

76 Sylvestre MP, Abrahamowicz M. Flexible modeling of the cumulative effects of time-dependent exposures on the hazard. Stat Med 2009:28:3437-53. doi:10.1002/sim.3701.

Appendix: Supplementary materials 